Sociologie et sociétés

SOCIOLOGIE

ETSOCIÉTÉS

\title{
Les impacts du cinéma américain sur le cinéma et la société québécoise
}

\section{Michel BRÛLÉ}

Volume 8, numéro 1, avril 1976

Pour une sociologie du cinéma

URI : https://id.erudit.org/iderudit/001110ar

DOI : https://doi.org/10.7202/001110ar

Aller au sommaire du numéro

\section{Éditeur(s)}

Les Presses de l'Université de Montréal

\section{ISSN}

0038-030X (imprimé)

1492-1375 (numérique)

Découvrir la revue

\section{Citer cet article}

BRÛLÉ, M. (1976). Les impacts du cinéma américain sur le cinéma et la société québécoise. Sociologie et sociétés, 8(1), 25-42. https://doi.org/10.7202/001110ar
Résumé de l'article

Le cinéma québécois n'est pas une industrie autonome. Le cinéma québécois dépend d'exploitants et de distributeurs qui ne sont pas québécois. Le cinéma québécois dépend pour sa production de capitaux qui viennent de l'extérieur du Québec et ce " non-Québécois " qui conditionne notre cinéma à ces trois niveaux est majoritairement américain. Ceci dit l'auteur essaie d'analyser l'impact qualitatif que le cinéma américain produit sur la société québécoise et sur l'industrie du cinéma au Québec. Ce problème cependant n'est pas particulier au Québec. " C'est à l'échelle internationale, y compris les pays de l'Est, que se pose le problème de l'impact du cinéma américain. Le cinéma, comme médium, est devenu américain ". Pourtant, et malgré tout, il y a dans le cinéma québécois des signes évidents d'une certaine volonté de garder ses distances vis-à-vis la production de type américain. 


\section{Les impacts du cinéma américain sur le cinéma et la société québécoise}

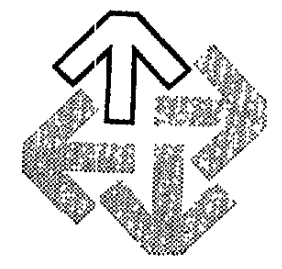

MICHEL BRÛLÉ

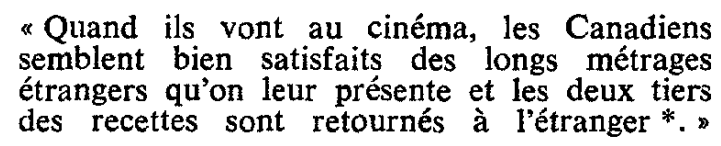

\section{DEUS EX CINEMA}

Il est aussi facile (ou difficile) à un sociologue québécois de démontrer l'impact du cinéma américain sur le cinéma québécois ou, plus largement, sur l'ensemble de la vie culturelle (au sens anthropologique) québécoise qu'à un catholique de démontrer l'existence de Dieu. Ce dernier a d'ailleurs un point commun avec le cinéma américain puisqu'il se «décompose » également en trois personnes (producteur, distributeur et exploitant) qui n'en font qu'une : l'industrie cinématographique et je dirai encore qu'à l'instar de Dieu, elle est partout et nulle part. Cette industrie du spectacle est en réalité fort discrète et préfère l'ombre des coulisses aux feux violents des projecteurs. Il faut faire preuve d'une bonne dose d'obstination pour avoir une vue quelque peu précise sur les multiples aspects de son fonctionnement et ses ramifications multidirectionnelles; ce qui rend particulièrement difficilc l'analyse de son impact.

* Georges-Emile Lapalme, extrait du premier rapport annuel 1968-1969 de la Société de développement de l'industrie cinématographique canadienne. 
En gros, je dirai qu'en matière de cinéma la situation du Canada vis-à-vis des États-Unis est assez particulière puisque le Canada est considéré comme faisant partie intégrante (et intégrée) du marché domestique américain. Dans son article ${ }^{1}$, Thomas H. Guback écrit :

The book value of American direct private investment abroad (largely branches and subsidiaries of American firms) in 1950 was reported to be $\$ 11.8$ billion. A provisionnal figure for 1973 set the value at $\$ 107.3$ billion of which $\$ 28.0$ billion was in Canada, twice as much as American investment in the world two decades earlier.

Ce type de rapport Canada-USA s'étend à beaucoup d'autres activités mais ce n'est pas là notre propos immédiat. Quant à la situation du Québec par rapport au Canada, et a fortiori par rapport aux USA, elle a cette particularité que tous connaissent : le Québec est majoritairement francophone ; ce qui n'empêche nullement le Québec d'être économiquement dirigé par des anglophones. En fait le Québec est économiquement et culturellement dominé.

Les deux coupures de presse qui suivent sont assez explicites; il n'est pas nécessaire d'y ajouter de commentaires.

La Presse, mercredi 6 août 1975 :

Ottawa - Les deux plus importantes chaînes de cinéma au pays, Famous Players et Odeon Theatres, ont accepté de présenter des longs métrages canadiens quatre (4) semaines par année dans chacune de leurs salles et d'augmenter leurs investissements dans la porduction de films canadiens. Le Secrétaire d'Etat, M. Hugh Faulkner, a précisé hier que le $\$ 1,7$ million que les deux chaînes ont accepté d'investir dans la production s'ajoutera aux quelques $\$ 3$ millions investis annuellement par la Société de développement de l'industrie cinématographique canadienne, un organisme gouvernemental.

$\mathrm{Au}$ cours d'une conférence de presse, le ministre a également annoncé qu'un nouveau règlement de l'impôt sur le revenu permettra à qui investit dans la production d'un film canadien, de déduire la totalité de son investissement, une mesure qui devrait, selon lui, stimuler l'industrie du cinéma au pays.

Le Jour, mercredi 6 août 1975 :

(...) - Cet accord passé entre Famous Players et Odeon d'une part et le Secrétariat d'Etat d'autre part est valable pour une période d'un an. Cet accord n'a pas force de loi.

(...) - Ce nouveau règlement de l'impôt sur le revenu est rétroactif au 18 novembre 1974.

\section{HIER ET AUJOURD'HUI}

Le découpage de l'objet a toujours été pour la sociologie un des problèmes majeurs, aussi voyons à quel découpage nous devons procéder pour traiter correctement de la relation entre le cinéma américain et le cinéma québécois dans une perspective sociologique.

1. Voir l'article de T.H. Guback dans ce numéro. 
On se dit tout d'abord que pour traiter un tel sujet la première chose à faire est cle connaître à fond toutes les données. Cela veut dire, puisque nous voulons traiter de la période 1945-1975, qu'il nous faut retracer 1. l'état de l'implantation de l'industrie cinématographique américaine au Canada et au Québec, et cela à un triple point de vue, soit au niveau de la production, de la distribution et de la consommation et 2 . avoir en main les données quant à la situation du cinéma canadien-français/québécois ${ }^{2}$ pour la même période.

Cela nous permet de savoir si ce cinéma était ou est encore dominant et, ce qui n'est pas négligeable, de pouvoir mesurer en quelque sorte cette domination. Cette démarche nous procurerait une image quantitative de la situation. C'est certes là un aspect intéressant du problème. Essayons de voir ce que cela donne.

$\mathrm{Du}$ point de vue de la production en 1945 deux longs métrages viennent d'être tournés au Québec, il s'agit de $\hat{A}$ la croisée des chemins de J.-M. Potevin et du Père Chopin, réalisé par Fédor Ozep, un Russe, naturalisé Français qui travaillait au Québec. Il n'y avait pas ni sur le territoire canadien ni au Québec de production américaine. En matière de réseaux de distribution nous savons quil y avait quelques cinémas indépendants et la mini chaîne de France-Film, tous les autres cinémas étaient entre les mains de capitaux anglo-américains.

$\mathrm{Du}$ point de vue de la distribution le seul distributeur canadien-français important était la compagnie France-Film ; les vrais réseaux de distribution appartenaient aux Anglo-américains. Quant aux salles, elles étaient, comme aujourd'hui, massivement la propriété des Américains et des Anglais. Les plus grandes salles de Québec et de Montréal appartenaient aux étrangers. France-Film possédait bien quelques cinémas et il $\mathrm{y}$ avait quelques indépendants mais on ne pouvait certainement pas parler d'une situation concurrentielle.

Le cinéma présenté au Québec était à toutes fins pratiques américain puisqu’à cause de la guerre les USA étaient les seuls à posséder une industrie florissante et quie les films des pays européens ne nous parvenaient pas.

$\mathrm{Si}$ nous regardons la situation des années 70 , on peut dire qu'elle ressemble étrangement à ce qui se passait il y a trente ans. Les réseaux de distribution sont toujours américains, les exploitants sont américains également et les indépendants s'approvisionnent dans la majorité des cas auprès des distributeurs américains. Quarit à la production il n'existe pas de lien entre le Québec et les USA. Le Québec a cependant développé une mini industrie cinématographique; mini si on la compare à celle qui est contrôlée directement ou indirectement par des capitaux américains mais importante si l'on tient compte de la population du Québec ( 6 millions).

Pourtant, d'une certaine manière, on ne saurait affirmer que l'industrie cinématographique américaine nous a nui ; du moins je ne crois pas que jusqu'aux années soixante nous ayions été très conscients de son emprise. Elle était là et c'était «normal», aussi «normal » que la présence massive de capitaux angloaméricains dans notre économie, aussi «normal » que l'exploitation de nos richesses naturelles par les multinationales américaines.

2. On a d'abord utilisé l'appellation * cinéma canadien * puis vers 1960 * cinéma canadien-français » et, enfin vers 1968, "cinéma québécois ». 


\section{DE L'IRRÉFUTABLE}

Toutefois, pour ce qui est de la présente étude, il m'apparaît secondaire de savoir si le cinéma américain a exercé au Québec une emprise pouvant se chiffrer à $68,3 \%$ ou à $72,1 \%$. Je ne nie pas pour autant l'importance et la pertinence de la recherche de données empiriques et la traduction de ces données en chiffres; de toutes façons, quelle que soit la statistique obtenue, il faudra toujours l'interpréter d'une certaine manière et dans un certain sens, d'où l'importance du découpage et de la perspective objective du chercheur.

Notre analyse, indépendamment de la valeur qu'on y attribuera, s'appuie sur de l'irréfutable: 1 . le cinéma québécois n'est pas une industrie autonome, 2 . le cinéma québécois dépend d'exploitants et de distributeurs qui ne sont pas québécois, 3. le cinéma québécois dépend pour sa production de capitaux qui viennent de l'extérieur du Québec (d'une manière immédiate en tout cas) et 4 . ce «non-Québécois» qui conditionne notre cinéma à ces trois niveaux est majoritairement américain. Enfin, on peut même ajouter en toute tranquillité et en toute certitude que le spectateur québécois a vu et voit encore plus de films provenant des États-Unis ou produits avec des capitaux américains que de toute autre provenance, l'Europe du marché commun comprise. Cette situation n'est pas nouvelle, elle a toujours existé. La seule chose qui soit changée c'est la conscience ou plutôt le début de conscience que les cinéastes et les cinéphiles prennent de cette domination.

Cette situation, si elle est neuve pour nous, n'a rien de particulièrement originale puisque nous nous retrouvons dans une situation semblable à celle que vivent plusieurs pays, autant ceux du tiers-monde que de pays industriellement avancés.

Il y a certes un important impact économique à cette situation, on évalue à $\$ 50$ millions environ les sorties d'argent engendrécs par cette domination. Il est donc tout à fait certain que nous contribuons fortement à financer le cinéma américain et partant notre propre domination. C'est un processus bien connu maintenant, une sorte de chaîne sans fin dont le résultat global est que les pays riches s'enrichissent de plus en plus que les pays pauvres s'appauvrissent de plus en plus.

\section{CHARYBDE ET SCYLLA}

Mais ce ne sont pas nécessairement les effets que l'on peut mesurer en pourcentage qui sont les plus nocifs. Ainsi, on pourrait présenter deux propositions, deux thèses qui en dépit de leur contradiction apparente me semblent justes.

Je crois en effet que si exploitants, distributeurs et producteurs américains décidaient d'investir massivement, ou du moins d'une manière significative, dans le cinéma québécois, il n'y aurait aucun changement structurel dans l'industrie cinématographique. Il y aurait tout simplement plus d'argent, donc moins de chômage dans ce secteur industriel. Par contre, il me semble tout aussi juste d'avancer l'énoncé contraire et de dire que la situation serait cent fois pire que celle que nous connaissons. 
Première thèse: il n'y aurait aucun changement structurel parce que d'ores et déjà une part importante de notre cinéma est conditionnée dans son imagination même par le cinéma américain, de type hollywoodien ou néo-hollywoodien. La raison de cet état de fait vient du fait que le cinéma mondialement dominant depuis la naissance du cinéma est américain et fabriqué comme un produit multinational ou transnational.

Seconde thèse : la situation serait pire car les importantes poches de résistance à un type de cinéma américain seraient très rapidement anéanties et il n'y aurait plus aucune possibilité objective de développer un cinéma différent. Les cinéastes producteurs d'un autre cinéma, s'ils tenaient à persévérer, devraient prenclre le maquis, entrer dans un véritable underground.

\section{BRÈVE HISTOIRE DU CINÉMA...}

ILe cinéma est le seul art né de la révolution industrielle, c'est un art né de la technique. Il faut se souvenir des origines de ce divertissement forain et de cette curiosité issue de la physique optique et de la chimie qui a suivi l'ascension de la bourgeoisie et des masses éduquées. Après quelques hésitations entre l'Europe et les États-Unis, il est devenu rapidement clair que les USA prenaient le dessus, à la fois au point de vue technique et au point de vue économique. On sait maintenan: que c'est après la première guerre mondiale et à cette occasion que le cinéma américain a pu prendre sur tous les autres cinémas nationaux un avantage irréversible, avantage qui s'est encore accentué après la seconde guerre mondiale. Les USA eurent tôt fait à cause de leur "know how » et de leurs moyens financiers - ce qui a permis ce que nous appelons aujourd'hui le «brain drain»de s'irnposer et d'imposer une forme de cinéma.

La grande victoire de la bourgeoisie fut de s'imposer à l'humanité comme étant l'Humanité et la grande victoire du bourgeois fut de convaincre, tantôt par la force tantôt par la ruse mais toujours en exploitant l'Autre, que lui seul incarnait l'homme naturel, que ses valeurs coïncidaient parfaitement avec les lois mêmes de la nature humaine. De même les Américains ont réussi à convaincre l'ensemble de la bourgeoisie qu'eux seuls incarnaient l'idéal de ce type d'homme qu'est le bourgeois. L' «American way of life» est devenu la norme de l'humanité. Que ce soit dans le secteur industriel, dans celui de la technologie ou de la science (y compris la science de la guerre) les USA ont dominé le monde et le dominent encore. Il n'est donc pas du tout étonnant que son cinéma occupe aujourd'hui $60 \%$ des écrans du monde. Ce serait la non-domination américaine dans ce secteur qui serait étonnante et non la situation que nous connaissons.

L'autre part nous commençons à connaître l'importance de l'imaginaire filmique dans la structuration des valcurs. Les industriels américains du cinéma et les hommes politiques ont reconnu très tôt la puissance de l'image et, plus que n'importe qui d'autres, ils ont saisi la force de $1^{\prime}$ "image innocente ${ }^{3} \gg$. En un sens il n'y a pas cinéma plus politique que le cinéma de divertissement, si l'on

3. L'image innocente, par exemple, est celle qui ne se présente que comme étant de l'ordre de nur divertissement, sans message, sans " contenu ". 
fait l'hypothèse que l'objectif politique est la dépolitisation des masses. La propagande est l'arme des faibles. La force des forts consiste à faire comme si, par exemple, la question politique était réglée. On ne fait pas un film didactique pour démontrer que les classes sociales n'existent pas, on raconte, l'histoire de quelqu'un qui, grâce à sa détermination, à son courage (individuel) et ses qualités, arrive à une situation «enviable » (i.e. qui obtient la richesse et le pouvoir). Donnons du pain et des jeux, se disait César ; donnons des images et du pop corn, ont dit les magnats du cinéma de type hollywoodien.

C'est à l'échelle internationale, y compris les pays de l'Est, que se pose le problème de l'impact du cinéma américain. Le cinéma, comme médium, est devenu américain.

Les USA ont gagné la bataille du cinéma grâce à la mise en place d'une stratégie financière qui leur a permis d'amener les divers pays consommateurs, comme les pays producteurs, à financer leur entreprise et par la production d'un vacuum vis-à-vis les réalités socio-politiques. Ce double processus a pour nom impérialisme; un impérialisme distrayant, agréable et dans un bon nombre de cas d'excellente qualité. Que la guerre est belle en technicolor sur écran géant!

\section{...ET DU QUÉBEC}

Le Québec avait une culture quand le cinéma est né, il avait une culture quand les capitaux étrangers se sont mis à s'intéresser à notre main-d'œuvre à bon marché et à nos matières premières à meilleur marché encore, mais nous ne le savions pas. Qu'a-t-on besoin de savoir que l'on a une culture, des traditions, une vision du monde, quand on est entre nous? Je ne veux pas remonter au déluge, cela n'est pas nécessaire, d'autant moins que notre déluge n'est pas très lointain.

Notre déluge à nous s'appelle la conquête (1760) et certains prétendent que notre arche de Noé fut l'Église. Peut-être aurais-je eu une autre opinion de la chose si j’avais vécu à cette époque là, mais maintenant que nous sommes passés au travers acceptons de bon gré ce que les autres (toujours les autres) ont nommé 《the priests ridden province». Certains de nos essayistes en ont été offusqués, peut-être avaient-ils oublié que ce clergé était le nôtre et que le renier relève davantage du politique que de l'analyse sociologique. Quand l'Autre, le Britannique, est arrivé il était vainqueur et il a agi comme tel, c'est-à-dire qu'il a pris en main les postes de commande et qu'il a commandé à sa façon. Nous nous sommes rendu compte qu'il n'était pas comme nous. Il parlait anglais, il ne croyait pas en Ia Sainte Vierge et il ne se confessait pas devant le curé, lequel d'ailleurs était marié. De plus, il différait par certains dons de la nature, car ce ne pouvait être des dons de Dieu - Dieu étant Français — du moins à l'époque. Parmi les dons qu'il avait reçus de la nature cet Autre en avait deux qu'il exerça à nos dépens ; il avait le don du commandement et le sens des affaires, bref c'était un matérialiste.

Devant cet état de fait sans appel nous nous sommes définis autrement. Nous nous sommes plongés corps et âmes, âme surtout, dans le culturel et spirituel, spirituel surtout. Nous parlions la plus belle langue du monde et plus que les 
Juifs encore - puisqu'ils avaient crucifié le Christ - nous étions le peuple élu de Dieu. Notre mission essentielle était d'évangéliser le reste du monde dont les Etats-Unis car nous étions les seuls catholiques-romains sur ce continent; l'anglophone étant nécessairement un protestant. Nous nous sommes donc réfugiés dans la culture, les arts libéraux et les professions encore plus libérales, laissant aux autres tout le champ de la technologie, de la science et des affaires.

Comme ce partage semblait l'affaire de l'Autre ${ }^{4}$ nous avons pu chacun de notre côté nous développer mais les deux lignes n'étaient pas absolument parallèles.

ILa terre ne pouvant pas les faire vivre, nos habitants s'exilèrent dans les villes et à l'étranger : aux États-Unis. Ils entrèrent d'abord dans les manufactures et les industries de transformation puis dans les services. Comme toute l'industrie appartenait à des capitaux anglo-saxons «nos gens» se retrouvèrent avec une culture qui n'avait plus de sens que dans le domaine privé. L'ensemble de la vie économique se passait selon les canons d'une culture et le reste de la vie était gouverné par une autre culture, sans qu'il y ait d'échanges véritables entre ces deux mondes.

C'est ce qu'a très bien expliqué Guy Rocher :

On peut dire que le Québec a vécu une étrange contradiction dans la première moitié du $20^{\circ}$ siècle : il adoptait les structures sociales de la civilisation industrielle, mais il gardait la mentalité, l'esprit et les valeurs de la société pré-industrielle. Il y a peu d'exemples d'autres sociétés en voie d'industrialisation à avoir connu une rupture aussi radicale entre les structures sociales et la culture ${ }^{5}$.

Cette schizophrénie sociale a duré jusqu'à la fin des années 50. Je partage entièrement l'analyse de Rocher et, comme lui, j'estime qu'il faut dépasser le constat d'une dichotomie entre les structures sociales et la culture et voir quelles ont été les médiations qui ont rendu supportable cet écart dramatique dans notre vie sociale.

Je pense que certains produits culturels, et particulièrement le cinéma, ont joué un très grand rôle dans notre acculturation réussie à la société nord-américaine.

\section{LA BÂTARDISE ET LA DIFFÉRENCE}

Reprenons les données essentielles. Nous sommes environ 5 millions de francophones en Amérique du Nord, soit 3\% de la population de cette partic nord du continent. Globalement nous venons tous d'un milieu rural et catholique et au cours du $20^{\mathrm{e}}$ siècle nous sommes projetés dans une civilisation industrielle que nous contribuons à créer mais dont nous ne sommes pas les moteurs. Nous sommes des exécutants et nous ne nous retrouvons chez nous qu'après le travail. Notre culture est une affaire de temps libre, ou plutôt de non-travail.

4. L'Autre ce fut d'abord l'Anglais d'Angleterre mais ce fut aussi l'Américain. On peut dire que depuis la dernière guerre ce sont essentiellement les Américains qui ont pris le contrôle de notre économie.

5. Rocher, Guy, Le Québec en mutation, Montréal, H.M.H., 1973, p. 18. 
Il n'y a pas de suspense à mon histoire puisque vous savez que nous avons survécu et que malgré tout nous sommes restés différents. Mais en même temps cette différence n'est pas de même nature que celle que nous pouvons constater chez les Français de France, ni d'ailleurs chez les Latino-américains. Car, quoi qu'on en dise nous ne sommes pas Français, ni Anglais, nous sommes des Nord-Américains parlant français. Dans notre cas appelons cela notre bâtardise mais une bâtardise que nous revendiquons. Nous prenons nos distances autant, sinon plus, à l'égard des Français que vis-à-vis des Américains. Au fond, si les Américains mangeaient mieux (c'est-à-dire comme nous), s'ils pensaient pas tout le temps en terme de biggest and the most nous nous sentirions plus à l'aise avec eux qu'avec nos fameux cousins de France qui essaient maintenant de nous raconter l'Amérique, à nous.

Donc nous sommes des francophones en Amérique. Et je suis tout à fait d'accord avec Rocher quand il écrit qu' « un autre élément de l'évolution culturelle récente, c'est l'imprégnation du Québec par la civilisation américaine ${ }^{6}{ }$.

Le Canadien-français des années ' 40 , comme celui des années ' 70 , n'a guère de contact direct avec les États-Unis. Certes, il va volontiers passer ses vacances au bord de la mer aux environs du Cape Cod ou encore en Floride l'hiver, mais il lui arrive plus souvent qu'autrement de rencontrer d'autres Canadiens-français, en vacances comme lui, que les Américains avec qui il communique vraiment. Ce n'est pas par ces brefs séjours dans le Maine, en Floride ou à New-York que le Québécois découvre les USA et les valeurs de la culture américaine. C'est chez lui, dans son foyer, devant sa télévision, en écoutant la radio et en allant au cinéma. Comme tous les habitants des pays industriellement avancés s'acheminant vers une ère post-industrielle, le Canadien-français est imprégné par les rythmes de la technologie et il s'est fait persuadé sans s'en rendre compte que la productivité et le rendement étaient les deux mamelles du progrès naturel, et de la civilisation.

Le Québécois n'a pas besoin de parler, ni de lire l'anglais ni même d'avoir mis les pieds aux «states», comme on dit chez nous, pour vivre à l'heure américaine, même si sa montre prend un peu de retard. La radio d'abord, par ses rythmes et ses chansons dont la majorité de la population ne comprenait pas les mots, le cinéma ensuite et enfin la télévision l'ont intégré au monde américain. Mais j'allais oublier la publicité ; elle est tellement omni-présente que nous ne la voyons plus, nous la respirons comme l'air ambiant. Et pourtant sa puissance est à la mesure de sa non-apparence.

Après avoir travaillé toute la journée, toute la semaine selon des cadences, des normes et une organisation fabriquées selon un modèle américain, le Québécois se reposait de son labeur en se laissant imprégner par des productions fabriquées de l'autre côté des frontières. Ainsi Jean-Baptiste qui n'était probablement jamais sorti de son village ou de sa petite ville, ou encore de son quartier s'il habitait Montréal, se détendait en regardant des images de New York ou de Chicago, le Texas ou le Grand Canyon bourré d'Indiens. À deux pas du Saint-Laurent, il se promenait sur le Mississipi au son des banjos.

6. Op. cit., p. 26. 
Après ce tour d'horizon, on peut maintenant circonscrire davantage non pas l'impact mais les impacts qu'eurent les films américains, d'une part, sur l'ensemble des spectateurs, qu'ils soient des simples consommateurs ou des cinéphiles avertis et, d'autre part, sur les artisans du cinéma québécois. Il est bien entendu que ce qui est valable pour l'ensemble du public l'est également pour les artisans.

\section{L'APPRENTISSAGE}

Par le cinéma ${ }^{7}$ le Québécois a pris contact avec des valeurs qui n'étaient pas les siennes. Il a appris l'importance du succès défini comme la réussite d'une compétition acharnée. Il s'est fait raconter les vertus de l'argent, indice numéro un de la réussite sociale et de la valeur d'un individu, et donc des sociétés puisque nous vivons dans une régime de libéralisme économique où ce qui est bon pour un individu ne saurait être mauvais pour l'ensemble de la société. C'est sous cette forme que le Québécois s'est fait vendre les mérites de la libre entreprise. Et si l'industrie a réussi à implanter la notion de productivité comme un absolu, le cinérna, aussi pernicieusement que la publicité, a pris à son compte le deuxième volet du développement économique de la société américaine, soit la «consommativité ».

Personne ne saurait nier l'impact qu'eut l'exhibition du niveau de vie américain tel que vu et corrigé par Hollywood et ses filiales. En projetant et en «spectacularisant» à travers le monde le «standard» de vie d'une certaine Amérique, en idéalisant les mille et un détails qui forment ce haut niveau de vie, l'industrie cinématographique a imposé au monde un étalon avec lequel chacun mesure son bien-être et sa relation avec cet idéal.

La fonction latente de la mise en valeur du mode de vie à l'américaine a été la plus vaste opération publicitaire jamais réalisée ... et réussie. Coca-Cola et General Motor pourraient en témoigner. Il faut bien voir également que cette mise en marché de «l'American way of life » s'est faite au détriment des modes de vie nationaux. Il suffit de lire les critiques et les comptes rendus de nos premiers longs métrages de la fin des années 40 pour comprendre le plaisir, la fierté et la fascination que ressentaient les Québécois quand il ont vu à l'écran leurs propres paysages, leurs coutumes et leur façon d'être.

Ainsi, à l'occasion du lancement de La Forteresse, écrivait-on :

(...) ce film a été complètement tourné dans les studios de Saint-Hyacinthe, dans la ville de Québec et aux chutes Montmorency.

Notre beau Canada français et les chutes Montmorency révélés au monde entier par La Forteresse ${ }^{8}$.

\section{A FRENCH CANADIAN DREAM}

Une revue exhaustive des coupures de presses de l'époque de notre premier cinéma dramatique montrerait clairement le type de réaction que nous eûmes. D'une part, on exprimait le plaisir et la fierté de voir enfin à l'écran des paysages

7. C'est évidemment une façon (rapide) de parler. Nous ne saurions penser que le cinéma soit l'unique pont entre la société américaine et la nôtre.

8. Le Dorchester, Sainte-Marie-de-Beauce, 11 mai 1949. 
de chez nous, de retrouver en images des coutumes et des façons d'être qui nous étaient propres ainsi qu'une langue qui était la nôtre (en opposition avec l'anglais, bien sûr, mais tout autant en opposition avec le français exotique de Paris).

Nous sommes done devant un vrai film de chez-nous. On y parle une langue fruste mais expressive, les images ne cherchent pas la virtuosité mais un réalisme simple. C'est exactement ce qu'il fallait et ce que le grand public appréciera. Nous avons aimé le film précisément parce qu'il ne cherche pas à éblouir, à épater, à imiter Hollywood ou Paris. (Léon Franque, La Presse, samedi $29 / 1 / 49$ ).

Il ne fait pas l'ombre d'un doute que cette affirmation du «cinéma canadien », comme on disait à l'époque, était aussi une négation du cinéma américain et européen.

D'autre part, il serait malhonnête de ne pas voir la fascination que le cinéma d'Hollywood exerçait sur notre conception de ce que devait être une industrie cinématographique. Le modèle demeurait le cinéma américain, c'est pourquoi nous cherchions malgré tout à bâtir une industrie pouvant rivaliser avec Hollywood. Devenir un Hollywood canadien-français, tel était notre plus secret désir.

Nous étions donc très ambivalents. Nous voulions voir notre réalité, notre monde et non celui des Etats-Unis ou de la France mais nous voulions aussi un produit semblable à celui qui nous parvenait de ces deux pays. Ce fut l'échec; car une fois passé l'effet de curiosité, les producteurs se sont retrouvés avec un sous-produit de cinéma américain ou européen. Ce n'est certes pas là la seule raison de l'échec de ce début du cinéma mais il est bien évident que le public formé, conditionné devrais-je dire, par le cinéma américain, n'a pas retrouvé dans les films québécois ce qu'il avait appris à avoir dans les films américains. Le cinéma québécois a cru pouvoir rivaliser avec les films étrangers en injectant un contenu local, sans toucher à la forme même de son produit. De plus, le côté nettement provincial des problématiques en cause rendait difficile ou même nettement impossible l'exportation de ces films. D'ailleurs même si nous avions produit des films exportables il aurait fallu passer par des distributeurs et des exploitants étrangers. Sachant ce que l'on sait aujourd'hui sur le contrôle monopolistique américain par rapport au cinéma, il est clair que cette entreprise ne pouvait pas réussir.

Néanmoins, le succès relatif qu'eurent ces premiers films tournés au Québec fut attribuable à l'effet de curiosité que suscite tout album de famille. Et le même phénomène se répéta au cours des années 60 quand, après un éclipse d'une quinzaine d'années, les films québécois réapparurent dans nos cinémas.

Une des critiques les plus sévères que l'on puisse adresser à l'industrie cinématographique USA implantée au Québec est d'avoir empêché ou, à tout le moins, retardé et rendu difficile l'expression d'une image de soi au cinéma. L'image de soi qu'un peuple peut voir au cinéma dépasse largement le simple reflet. On oublie trop souvent, et cela tient au médium cinéma comme tel qui produit cette impression extrêmement forte de réalité, que l'image est une production ou une construction, au même titre que l'histoire racontée par le film. Tout film est une 
interprétation de la réalité existante et comporte un projet d'exister. J'irais même jusqu'à dire que le film traduit moins ce qui existe déjà que ce que les hommes cherchent à faire devenir ou à maintenir - ce qui est aussi une sorte de projet d'avenir. C'est ce dont les Québécois ont été privés, du moins jusqu'à tout récemment. Alors que depuis 1960 la chanson a réussi l'extraordinaire tour de force d'arriver à chanter la collectivité québécoise et son projet social, le cinéma a été grandement paralysé dans sa tentative de produire des images et des rêves pour: les hommes d'ici. Il y a bien un cinéma qui travaille dans cette veine mais il faut bien avouer qu'il est minoritaire et qu'il n'a pas pu encore s'imposer dans les grands circuits commerciaux.

\section{UNE CERTAINE VIOLENCE}

Sans vouloir ajouter un chapitre aux centaines d'ouvrages et d'articles consacrés au thème de la violence au cinéma, disons seulement que la violence a occupé et qu'elle occupe encore une place prépondérante sur les écrans. Le cinéma américain a réussi à créer une esthétique de la violence et à imposer au monde sa conception de la violence, un certain type de violence, soit une violence physique, spectaculaire et presque toujours individuelle. Il n'est guère question de violence politique, économique et sociale qui atteint les hommes dans leurs âmes et leur raison de vivre; et quand par exception nous retrouvons ce type de violence, un individu sort de l'ombre et mène son propre combat et arrive seul à vaincre les forces du mal. Pour des raisons historiques, socio-économiques et culturelles la violence a connu aux États-Unis un développement généralisé et d'une grande amplitude. Et il est à remarquer que si le cinéma montre beaucoup de violence, il ne montre pas les antagonismes structuraux qui existent entre les classes sociales, concept, incidemment, peu prisé par la sociologie américaine. La violence américaine au cinéma me semble donc fortement idéologisée et, comme tout discours idéologique, cette violence est une interprétation de la société et vise à un certain type d'existence de cette société.

Or cette violence ou ces types de violence véhiculés par le cinéma américain étaient tout à fait étrangers à l'expérience canadienne-française et, encore aujourd'hui, en dépit d'une augmentation certaine du taux de criminalité, nous ne pouvons pas affirmer connaître cette violence qui règne dans presque toutes les grandes villes américaines. Le Canada comme tel et le Québec en particulier sont très * en retard » par rapport au phénomène de la violence urbaine, par exemple. Même à cet égard nous sommes différents. Je ne suis pas en mesure d'évaluer l'impact de cette violence cinématographique sur la société québécoise et ce n'est pas là mon propos. Une chose est certaine la violence présentée dans les films américains est en grande partie déconnectée de l'expérience du spectateur québécois.

On pourrait passer en revue tous les secteurs de la vie et montrer comment ce que le spectateur reçoit ne correspond pas à son expérience culturelle. Pourtant même si cette affirmation est fondamentalement exacte, il n'en demeure pas moins que le cinéma a exercé chez les Canadiens-français une véritable fonction éducatrice. Bien qu'il n'existe pas d'études sur le sujet, je croirais volontiers que ce cinéma a largement contribué à faire entrer les Québécois dans des probléma- 
tiques urbaines et industrielles et qu'à cet égard (quel que soit le jugement de valeur que l'on porte sur cette initiation) le cinéma américain rejoignait les gens en leur posant des problèmes qu'ils n'avaient pas eu encore à résoudre mais qui allaient bientôt se poser dans tous les pays industriellement avancés. Il ne s'agit pas de prophétie ou de hasard. La technologie étant essentiellement américaine il était fatal que tôt ou tard tous ceux qui participent au développement industriel connaissent des problèmes similaires. Où le cinéma américain a joué un rôle que je qualifierais de pernicieux c'est quand après avoir posé le problème, il «impose » ses solutions et ses propres réponses; mais ça aussi doit être analysé.

\section{L'ÉCHEC DU HOLLYWOOD CANADIEN-FRANÇAIS}

La réalité de cet impact du cinéma américain étant extrêmement complexe, il faut, je crois, revenir à la fin des années 40 pour essayer de saisir sur le vif, dans une sorte de description phénoménologique, ce qui se passe quand un cinéma national entre soudainement en compétition avec un cinéma monopolistique. Très schématiquement nous tenterons de cerner la situation de la manière suivante.

Il est bien évident qu'avant les premiers films canadiens il n'y avait que des films étrangers dont la très grande majorité venaient des USA. Ce cinéma occupait toute la place. On peut même facilement dire que le cinéma américain était tout simplement LE cinéma et des générations de Québécois s'étaient initiés au septième art à son contact.

Mais voilà qu'arrivent sur le marché des films fabriqués ici, avec des comédiens d'ici, des images d'ici, une langue d'ici et des histoires made in Québec. La représentation de cet «ici » est cependant, par naïveté et par manque de métier, un peu grossière, mal équarrie, avouons-le. Ces films louchent par certains aspects vers un passé que nous qualifierons de traditionnel. Quant aux comédiens, ils en sont forcément à leurs premiers films et ils viennent pour la plupart de la radio et du théâtre.

D'autre part, il y a le cinéma américain avec des comédiens qui ne sont pas d'ici mais qui, grâce à la publicité et à toute l'industrie du «star system», sont aussi connus que les vedettes locales. Quant aux scénarios ils ont vraiment peu à voir d'une manière immédiate avec ce que sont les Canadiens-français. Néanmoins, faisons l'hypothèse farfelue que tous les cinéphiles délaissent dans un premier temps ce cinéma et qu'ils s'engouffrent dans les salles où l'on projette du cinéma québécois.

La première réaction à l'égard de ce nouveau cinéma en fut une de curiosité et de fierté. Le public pouvait enfin voir ses vedettes dont il ne connaissait que la voix pour avoir suivi pendant des années les radio-romans. Il y avait aussi la dimension paysage. La critique d'ailleurs mit en valeur cet événement ; enfin on voyait les chutes Montmorency et nos belles Laurentides. Quant à l'histoire que racontait le film, on en soulignait d'abord les aspects folkloriques, l'illustration de nos coutumes ou de nos manières d'être. L'intrigue était fort simple et se prêtait bien aux scènes pittoresques. La critique pour sa part souligna avec force la naissance d'une nouvelle industrie et prophétisa la création d'un Hollywood francophone. 
On disait que chaque nouveau film était meilleur que le précédent et qu'on s'acheminait lentement mais sûrement vers l'idéal américain. Au début des années '50 et par la suite, les nouveaux critiques formés à l'école des ciné-clubs levèrent le nez sur l'ensemble de la production de cette époque et, à vrai dire, en eurent honte car elle ne pouvait pas selon eux soutenir la comparaison avec les films américains ou européens. Ce jugement sans nuance sur le cinéma de cette époque a encore cours aujourd'hui et il est de bon ton de ridiculiser les films de cette période. Pourtant une analyse sérieuse de ces films mène à des conclusions beaucoup plus nuancées, sinon tout à fait opposées. Les travaux que j'ai faits sur ces films montreront que ce cinéma racontait essentiellement le passage ${ }^{9}$ d'une société traditionnelle à une société industrielle et que ce cinéma traitait d'une façon très précise de l'inanité des valeurs d'une culture coupée des nouvelles structures économiques. Il semble bien cependant que la réaction au contenu manifeste ait été plus forte ou mieux comprise que le contenu structurel. Ces films en réalité empruntaient le décor et les atours de l'idéologie traditionnelle pour en faire une critique sévère et sans appel. Cette forme créa, je crois, un malentendu et ces films passèrent pour une défense et une illustration du Canadien-français catholique, modèle 1890 .

Une fois la curiosité satisfaite, soit après avoir vu avec émerveillement les chutes Montmorency, après avoir vu et reconnu les talents locaux et suivi avec un maximum d'intérêt une histoire qui se passe dans $90 \%$ des cas en milieu rural ou quasi rural, les spectateurs durent se trouver devant un manque. C'est-à-dire qu'ils retrouvèrent sous une forme dégradée et artisanale un produit qu'ils avaient déjà consommé dans sa forme "authentique »: décors grandioses, acteurs professionnels de la caméra (et non de la radio comme c'était le cas ici), histoires construites par des spécialistes, dialoguistes chevronnés, etc. De plus, et c'est là à mon avis l'élément le plus important, le film américain n'était pas déconnecté par rapport à l'expérience du travailleur industriel en milieu urbain. Béla Balazs disait en substance ${ }^{10}$ que le cinéma comme art était le produit d'une société industrielle capitaliste et, comme Balazs, je crois que le prototype a été fabriqué à Hollywood. Les westerns relèvent autant de lindustrialisation et de l'urbanisation que les films policiers.

Donc, alors que le cinéma américain parlait ville et milieu industriel, le film québécois de la période '43-'53 évoquait encore, même si c'était pour s'y opposer, la « vraie vie »du Québécois. Mais cette «vraie vie » était déjà dépassée, ses racines n'étaient plus alimentées que par les discours des anciennes élites et elle n'était revécue que dans la vie privée à l'occasion de fêtes. Ce que le spectateur moderne de 1949 voyait à l'écran était lié à une problématique ancienne déjà, mais non culturellement liquidée. La culture traînait de la patte, elle ne s'était pas encore mise aux nouveaux rythmes de la vie économique du temps de guerre et l'après-guerre.

9. Voir dans ce numéro notre analyse d'Un homme et son péché et de Séraphin.

10. Voir Theory of the film. Character and Growth of a New Art, London, Dennis Dobson Ltd., 1950. 
D'autre part, le cinéma américain ne touchait pas non plus la «vraie vie 》 des Canadiens-français ; il avait l'air, comme on dit, mais pas les paroles. Le monde que le film américain présentait n'était pas encore la réalité des Québécois, mais ces derniers sentaient obscurément par leur expérience du travail et de la vie économique soumise à la technologie, que leur vie irait en ressemblant à ce qu'ils entrevoyaient sur les écrans de la Famous Players.

D'un côté la culture, une culture un peu passée et dépassée ; de l'autre, les structures économiques, c'est-à-dire quelque chose en plein devenir. Or entre ce qui n'est pas encore tout à fait fini et quelque chose qui n'est pas encore tout à fait arrivé, le Québécois ne retrouve pas son présent au cinéma. Aussi en 1952 il s'empressera d'acheter un téléviseur et il tentera d'amortir son investissement en accumulant les heures d'écoute. Car la télévision est essentiellement un médium du présent.

\section{DE LA MORALE À LA CRITIQUE ÉCONOMIQUE}

Les gens du cinéma ont eux été doublement atteints par les retombés de la présence du cinéma américain et européen. Ils ont été marqués comme tout le monde par ce que nous venons de décrire et ils ont eu à vivre en tant qu'artisans du cinéma cette réalité «étrangère ». Je mets étrangère entre guillemets parce que ce n'est que tout récemment (une douzaine d'années tout au plus pour certains) que les cinéastes ${ }^{11}$ ont pris conscience que ce cinéma et cette industrie leur étaient étrangers.

Passons rapidement par dessus les conséquences les plus visibles de l'implantation de l'industrie cinématographique américaine au Québec. Par conséquences les plus visibles, j'entends le fait que la très grande majorité des circuits sont directement ou indirectement contrôlés par les Américains et que ces derniers ne furent jamais intéressés ni à distribuer ni à programmer les films produits ici. Même l'agence gouvernementale canadienne du cinéma, l'Office national du film, doit passer par Columbia Picture pour distribuer ses films. Quant aux investissements dans la production, c'est tellement marginal et récent que ça ne vaut pas la peine d'en parler.

La maturation ${ }^{12}$ des cinéastes québécois pourrait se résumer de la façon suivante. Il y eut d'abord l'étape de la formation des ciné-clubs et des premières revues de cinéma. On y traitait des différentes techniques cinématographiques dans une perspective didactique et on y faisait de la critique. C'était une critique très impressionniste et fort moralisatrice. On confond inconsciemment cinéma et vie et on porte sur les films des jugements moraux que l'on porterait sur des événement ou des hommes. Néanmoins ce travail fait dans le cadre des ciné-clubs contribue grandement à développer un goût, voire une véritable passion pour la chose cinématographique. À la fin des années 50 et au début des années 60 on constate que plusieurs des militants de la première heure se sont intégrés dans divers secteurs de la production cinématographique, notamment dans le cadre

11. Par cinéastes, j'entends ici aussi bien les monteurs, les caméramen, les ingénieurs du son que les réalisateurs. L'histoire a en effet démontré que plusieurs cinéastes en sont venus à la réalisation après avoir exercé ces divers métiers.

12. Je parle de maturation car il faut garder en mémoire que nos plus vieux cinéastes, à quelques exceptions près, n'ont pas encore cinquante ans. 
de l'Office national du film. La relève est assurée par une équipe de jeunes afficionados de l'écran, la plupart sont étudiants à l'université. Cette deuxième étape est surtout marquée par l'attention toute particulière que l'on porte sur les films québécois (anglais et français) qui sortent de l'O.N.F. C'est une période extrêmement dynamique où chaque court métrage qui sort de cette boîte est analysé et discuté fébrilement. Toutefois, le gros débat de cette période demeure la censure. Le Québec est reconnu pour avoir un bureau de censure extrêmement sévère. L'ère de la révolution tranquille est commencée et dans tous les secteurs de la vie sociale, le Québec, avec son nouveau gouvernement libéral, essaie de rattraper le retard accumulé au cours des années de règne de l'ancien premier ministre M. Duplessis. Il y a un fossé entre ce que les gens sont devenus et les cadres juridico-politiques dans lesquels ils doivent vivre. La grande bataille de la censure est un moment important de l'histoire du cinéma mais c'est aussi à l'échelle de la société globale une sorte de tournant historique. Ce bureau de censure constituait une sorte de bastille de conservatisme et du puritanisme. C'est surtout autour des films français que se fit la bataille ${ }^{13}$. Ces combats contre la censure ont drainé une très grande énergie intellectuelle. La troisième phase a été centrée sur la nécessité d'une loi-cadre ou, autrement dit, sur la nécessité pour le gouvernement d'intervenir et de légiférer dans ce secteur de l'industrie culturelle. Cette phase qui a duré un douzaine d'années vient à peine de se terminer par la mise en place d'une loi-cadre au cours de l'été dernier.

Cette lutte pour obtenir que l'État intervienne pour protéger et favoriser le développement de l'industrie cinématographique s'est faite à partir de dossiers où il a bien fallu que les cinéastes analysent leur situation objective. C'est en faisant ce travail qu'ils purent mettre en relief l'impérialisme américain en matière de cinéma et la dépendance du Québec sous ce rapport. Il faut bien voir cependant que cette démarche des cinéastes coïncidait avec des démarches similaires dans les différents secteurs de l'activité socio-économique. Bref, le travail que les artisans du cinéma faisaient était en synchronie avec ce qui se faisait dans toute la société québécoise. Rétrospectivement il est facile de constater qu'un grand nombre d'analyses tournaient autour des thèmes de l'aliénation ct de la colonisation du peuple québécois, thèmes dominants d'une certaine analyse critique du Québec.

C'est pendant cette période qui va grossièrement de 1964 à maintenant que s'est développé vraiment le cinéma québécois. Au cours de ces années près de 250 longs métrages sont produits, la plupart depuis 1968, moment où la Société de céveloppement de l'industrie cinématographique canadienne (SDICC) est entrée en action ${ }^{14}$.

La production s'est organisée autour des secteurs privé et gouvernemental. Mais ni le secteur public ni le secteur privé ne sont monolithiques. Dans les deux secteurs on retrouve des films d'auteurs et des films à vocation parfaitement commerciale. Ceci s'explique du fait que la société québécoise est petite, qu'il y a beaucoup d'interactions entre les créateurs et que l'ONF n'a jamais eu vraiment de politique en matière de longs métrages.

13. Le Rideau cramoisi, d'Alexandre Astruc et surtout Hiroschima mon amour, de Resnais furent entre autres les grandes occasions de ces batailles.

14. Cette société d'Etat comme son nom l'indique a pour mandat d'aider financièrement la production de films canadiens. Son budget était de dix millions en 1968. 


\section{MALGRÉ TOUT}

Le cinéma québécois se caractérise jusqu'à maintenant par une référence constante aux problèmes que la société québécoise vit comme totalité. Ceci est vrai pour notre cinéma le plus commercial et a fortiori pour le cinéma dit d'auteur. Bien sûr, les références à la société québécoise comme entité n'ont pas dans tous les films la même profondeur ni la même richesse, mais il y a comme un lien organico-culturel entre les producteurs artistiques et la société québécoise. Marcel Rioux $^{15}$ se plaît à dire que le Québec est «tricoté serré ». À vrai dire la distinction entre le cinéma commercial et cinéma d'auteur est artificielle même si chacun pense comprendre ce qu'on cherche habituellement à exprimer par cette distinction. Tout cinéma commercial ou plutôt tout cinéma s'inscrit dans un circuit micro ou macro industriel. Dans l'état actuel des choses, plus le budget d'un film est considérable plus les liens qui attachent les artisans du cinéma au capital sont grands et plus le produit risque d'être politisé par les intérêts des bailleurs de fonds qui, eux, n'ont pour but que de maintenir en place un système qui rapporte beaucoup d'argent. Et c'est là que réside la différence entre l'origine des capitaux. Quand l'argent provient de l'État l'accent est mis sur la protection du système et quand il provient du secteur privé l'accent est mis sur le profit. Quant au rôle d'une société d'État comme la Société pour le développement de l'industrie cinématographique canadienne il est mixte car cette société joue ouvertement sur les deux tableaux à la fois. Il n'y a qu'à analyser le questionnaire auquel doivent répondre ceux qui analysent les scénarios pour la S.D.I.C.C., pour se rendre compte du type de film souhaité et valorisé par la S.D.I.C.C. : un cinéma narratif axé sur la psychologie des personnages et sur l'universalité des sentiments. Et quand il est question du milieu, c'est en référence non pas à la société ni aux groupes en lutte dans cette société mais en fonction des anachronismes à éviter ou des erreurs techniques à prévenir. Il est bien évident que le gros argentier du cinéma québécois (et canadien) a aligné ses critères et sa conception même du cinéma d'ici sur l'industrie américaine.

Quand on sait qu'il est à peu près impossible de faire un film sans le concours de cette Société, on voit quelle pression est exercée sur le cinéma québécois. Comme tous les autres cinémas il est poussé à jouer le rôle de «valium culturel 》 et de moyen d'évasion d'une société dite de loisirs (à crédit: 22\% par année) où pour survivre il est paradoxalement recommandé d'aller s'exciter devant un film de sexe, de se faire peur avec des films d'horreur ou de se faire chatouiller par des films comiques ; n'importe quoi, sauf la réalité.

Malgré tout, nous demeurons optimiste parce que jusqu'à maintenant la marginalité des Québécois par rapport à l'ensemble de la société nord-américaine les a toujours servis et qu'elle les a protégés des contradictions exacerbées de cette société et, deuxièmement parce que l'art exerce toujours aussi une fonction hautement critique dans nos sociétés industrielles.

15. Rioux, Marcel, la Question du Québec, Paris, Seghers, 1969. Les Québécois, Paris, Ed. du Seuil, 1974. 


\section{RÉSUMÉ}

Le cinéma québécois n'est pas une industrie autonome. Le cinéma québécois dépend d'exploilants et de distributeurs qui ne sont pas québécois. Le cinéma québécois dépend pour sa production de capitaux qui viennent de l'extérieur du Québec et ce "non-Québécois " qui conditionne notre cinéma à ces trois niveaux est majoritairement américain. Ceci dit l'auteur essaie d'analyser l'impact qualitatif que le cinéma américain produit sur la société québécoise et sur l'industrie du cinéma au Québec. Ce problème cependant n'est pas particulier au Québec. * C'est à l'échelle internationale, y compris les pays de l'Est, que se pose le problème de l'impact du cinéma américain. Le cinéma, comme médium, est devenu américain ». Pourtant, et malgré tout, il y a dans le cinéma québécois des signes évidents d'une certaine volonté de garder ses distances vis-à-vis la production de type américain.

\section{SUMMARY}

The movie industry in Quebec is not an autonomous industry. It depends upon exploiters and distributors who are not from Quebec. It is dependent for its capital which comes from outside and the "non-quebeckers" on which it depends and which condition it at these three levels are in majority american. Having said this the author trys to analyze the qualitative impact which American films produce in Quebec society and on the film industry in Quebec. This problem is however not specific to Quebec. "The impact of american films is a problem on a world wide scale, including the countries in the East. The film, as a medium, has become american". Nevertheless, and inspite of everything, there is evidence of a certain will to maintain a distance from American type productions in the Quebec film industry.

\section{RESUMEN}

El cine quebequense no es una industria autónoma. El cine quebequense depende de los dueños de las salas y de los distribuidores que no son quebequenses. El cine quebequense depende para su producción de los capitales que vienen del exterios de Quebec y este " no quebequense "que condiciona nuestro cine a esos tres niveles es mayoritariamente americano. Dicho esto el autos trata de analizar el impacto calitativo que el cine americano produce sobre la ciudad quebequense y sobre la industria cinematográfica en Quebec. Ese problema sin ernbargo no es particular de Quebec. "Es en la escala internacional, incluidos los paises del este, que se plantéa el problema del impacto del cine americano. El cine como medio se ha vuelto americano ». No obstante, y a pesar de todo, hay en el cine quebequense signos evidentes de una cierta voluntad de guardar sus distancias frente a la producción de tipo americano. 